\title{
Design of a Customized Neck Orthosis for FDM Manufacturing with a New Sustainable Bio-composite
}

\author{
Rita Ambu ${ }^{1(凶)}$, Alessandro Motta ${ }^{2}$, and Michele Cali ${ }^{3}$ \\ ${ }^{1}$ Department of Mechanical, Chemical and Materials Engineering, \\ University of Cagliari, via Marengo 2, 09123 Cagliari, Italy \\ ambu@unica.it \\ 2 Studio di Radiologia Motta S.r.l., \\ Via Francesco Crispi 4, 95032 Belpasso, CT, Italy \\ alemotta.med@gmail.com \\ ${ }^{3}$ Electric, Electronics and Computer Engineering Department, \\ University of Catania, Viale A. Doria 6, 95125 Catania, Italy \\ mcali@dim.unict.it
}

\begin{abstract}
The interest in developing customized external orthopaedic devices, thanks to the advent of Additive Manufacturing (AM), has grown in recent years. Greater attention was focused on upper limb casts, while applications to other body's parts, such as the neck, were less investigated. In this paper the computer aided design (CAD) modelling, assessment and 3D printing with fused deposition modelling (FDM) of a customized neck orthosis are reported. The modelling, based on anatomic data of a volunteer subject, was aimed to obtain a lightweight, ventilated, hygienic and comfortable orthosis compared to the produced medical devices generally used for neck injuries. CAD models with different geometrical patterns, introduced for lightening and improving breathability, were considered, specifically, a honeycomb pattern and an elliptical holes pattern. These models were structurally assessed by means of finite elements analysis (FEA). Furthermore, an innovative composite material was considered for 3D printing. The material, Hemp Bio-Plastic ${ }^{\circledR}$ (HBP), composed by polylactic acid (PLA) and hemp shives, offers different advantages including lightweight, improved superficial finish and antibacterial properties. The results obtained in terms of design methodology and manufacturing by $3 \mathrm{D}$ printing of a prototype have shown the feasibility to develop customized cervical orthoses, with potentially improved performance with respect to cervical collars available on the market also thanks to the use of the innovative composite material.
\end{abstract}

Keywords: CAD $\cdot$ Neck orthosis $\cdot$ Additive Manufacturing $\cdot$ Bio-composite

\section{Introduction}

Additive Manufacturing (AM) techniques are increasingly considered as a viable manufacturing method for various applications. In fact, AM enables the production of complex geometries that were previously difficult or impossible to manufacture [1]. 
In particular, the area relative to biomedical applications has some relevant potential benefits of using AM. These include tissue engineering bone scaffolds [2-4], preoperative planning 3D printed models [5, 6], patient specific instruments [7], and customized orthopaedic devices [8]. The latter involves the customized design and AM printing of external medical devices, or orthoses, used in the treatment of specific pathologies. An orthosis is generally designed to stabilize, immobilize, prevent deformity or protect against injury. The fabrication of a customized device can be, potentially, more advantageous than that of a prefabricated orthosis, both clinically and for the patient's comfort.

Recent research on personalized orthoses was primarily focused on upper limb casts [9-18] highlighting the feasibility of AM in this field. On the other hand, few investigations [19] have considered this manufacturing method as a viable option for the cervical region. This can be attributable to the complex functionality of a cervical orthosis which has to support, prevent immobilize or correct the spine. Moreover, the clinical aspects have to be combined with other factors such as the sensation of comfort and the aesthetic appearance.

In this paper, the design and manufacturing with fused deposition modelling (FDM) of a customized orthopaedic neck immobilization device are reported. The aim of the study is to evaluate the customized design and 3D printing of a neck orthosis highlighting the potential benefit of this methodology. Semi-rigid cervical collars are available on the market to protect the cervical spine after injuries and, postoperatively, to immobilize the spine following surgical reconstruction. A primary measure of their effectiveness is the capability to immobilize the spine. As known, some problems are usually associated with the use of these devices including muscle atrophy, skin rush or breakdown, pain, lack of hygiene, odor, heaviness and discomfort.

The design of the customized cervical orthosis was, therefore, conceived for an optimal performance by adopting a proper geometry for functionality, and, at the same time, reducing weight and improving breathability for a better comfort. Furthermore, the concept of sustainability, in terms of material, was introduced by considering for $3 \mathrm{D}$ printing an innovative composite material. The new composite is a $3 \mathrm{D}$ printing filament composed by polylactic acid (PLA) and hemp shives, patented by MICA s.r.l. (Ragusa-Italy) as Hemp Bio-Plastic ${ }^{\circledR}$ (HBP). The sustainable nature of the filament, its lightweight, improved mechanical performance [20] and superficial finish, and last but not least its antibacterial properties [21] were the main reasons for this choice.

The study was conducted starting from computer tomography (CT) scans of the anatomic area of the neck made available by a volunteer subject, that were used to reconstruct the part of interest. The next step consisted in the computer aided design (CAD) modelling of the customized cervical orthosis based on the reconstructed neck. Different geometrical patterns were introduced in the CAD model for lightening the structure and improving breathability. The models obtained were numerically assessed by means of Finite Elements Analysis (FEA) in order to globally evaluate the proposed geometrical configurations. Finally, a prototype of the designed cervical orthosis, made with the advanced composite, was manufactured with fused deposition modelling. 


\section{Design Procedure}

The first step in the design procedure was relative to the acquisition and digital reconstruction of the neck (human neck). A digital representation of an outer part of the human body can be obtained by means of 3D photogrammetric or laser scanners. However, a 3D reconstruction can also be achieved from Computer Tomograpy (CT) scan acquisitions, if available. For this study, CT scan acquisitions (Fig. 1) were provided by a volunteer subject which underwent a CT Scan for personal issues.

These data were used to create the neck 3D model. Through the use of the Materialise's Interactive Medical Image Control System (MIMICS) software, the CT images (about 600 each analyzed case) were filtered to eliminate the parts that were not used (such as bones, internal organs, or clothes).

In particular, the "thresholding" filter was used for a first definition of the segmentation. The object was defined based on lower and upper thresholds. In the first case, the object of the segmentation had all the pixels in the images with a value which was greater than or equal to the threshold value; in the second case, the pixel value had to be comprised between both the threshold values to be part of the segmentation object.

The CT images are a pixel map of the linear attenuation coefficient of the X-rays in tissue. The pixel values were reduced so that the linear attenuation coefficient of the $\mathrm{X}$-rays in air was equal to -1024 and that of water was equal to 0 . This scale is called "Hounsfield scale" from the name of one of the tomography pioneers, Godfrey Hounsfield. The calculation of the polylines was made as the contours of the pixels in the axial view, so they were built automatically for the cavities inside the mask, as well.

In order to delete such cavities, considering the final interest in extrapolating the three-dimensional model only of the outer geometry of the neck, the "Cavity Fill From Polylines" filter was used, thus obtaining a new mask.
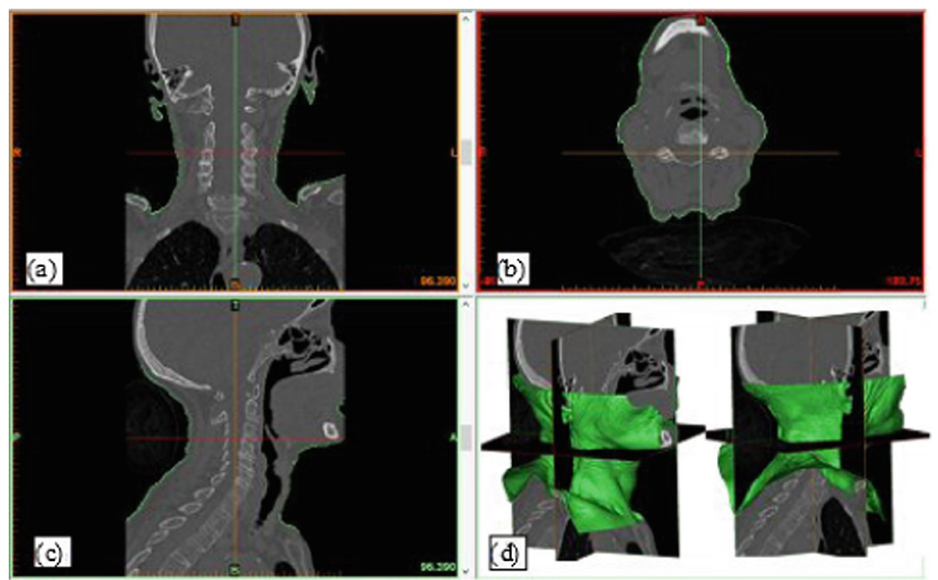

Fig. 1. CT Scans views: (a) frontal; (b) transversal; (c) lateral; (d) isometric. 


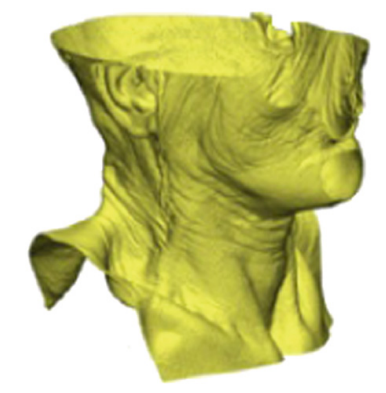

Fig. 2. 3D tessellated final surface.

Once the geometry was "cleaned", the part associated with the mask ("Calculate Part" operation) was calculated (Fig. 2). This part is a mesh of the neck in STL format.

This model was imported in a CAD environment and the automatically reconstructed surface was used to design the geometry of the neck orthosis.

The CAD model developed was intended to design a device with functionality similar to that of cervical semi-rigid collars available on the market. Generally, this kind of cervical collar is conceived as an orthosis that fits the neck, anywhere from the jaw to the chest. It is used to restrict movement and support the neck to allow healing when treating a cervical injury, fracture, or surgical procedure. On the other hand, soft cervical orthoses, which have a lighter geometrical design, help in the treatment of minor neck strains, support the neck and, control pain after an injury (e.g., whiplash).

For obtaining the model, subsequent sections of the neck were reconstructed, also introducing an offset with respect to the neck surface in order to allow the placement of a soft pad in the inner part of the orthosis to improve the comfort. Then, the surface obtained was thickened $(4 \mathrm{~mm})$ to obtain the ultimate architecture. The value of the thickness chosen was the average thickness usually adopted in prefabricated orthoses. A geometrical pattern was finally introduced in the obtained model.

In fact, as stated in the introduction, among the problems usually associated with the use of a cervical orthosis, there is the lack of breathability which can result in discomfort. Furthermore, since the orthosis needs be worn in a very visible part of the body, the aesthetic aspect is also relevant. Thus, the introduction of a pattern on the customized orthosis has several purposes, in addition to lightening the structure which is one of the main advantages allowed by the use of additive manufacturing.

Different patterns were chosen to fill the modelled structure. The first geometry considered was a honeycomb pattern with uniform cells. The cell size regularity allows to attain a more isotropic mechanical behaviour [22] with respect to a non-uniform pattern, such as a Voronoi pattern, frequently used for AM orthoses. A Voronoi pattern was also taken into account for this application; however, after a preliminary analytical analysis it was decided to discard this pattern since the relative model showed a low stiffness with respect to the stress that the neck orthosis has to withstand. 
A zone devoid of the pattern was also provided to insert hinges for wearing purposes. The finally obtained honeycomb pattern orthosis, shown in Fig. 3, has a volume fraction of 0.79 . This last parameter was chosen to globally characterize the geometrical pattern in terms of porosity, which can affect the structural performance of the orthosis.
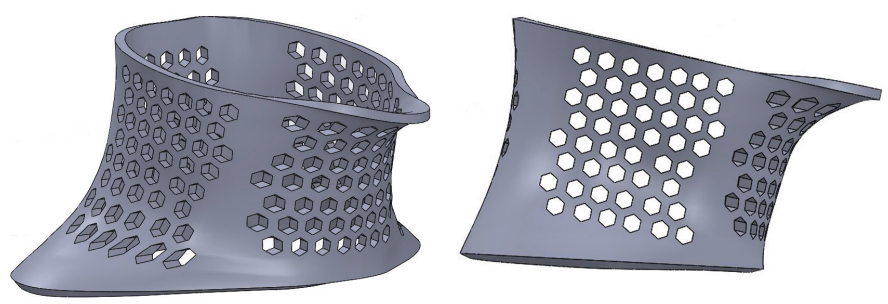

Fig. 3. CAD model of the honeycomb pattern cervical orthosis.

The other geometrical pattern considered, shown in Fig. 4, consisted of elliptical holes oriented at $\pm 45^{\circ}$.
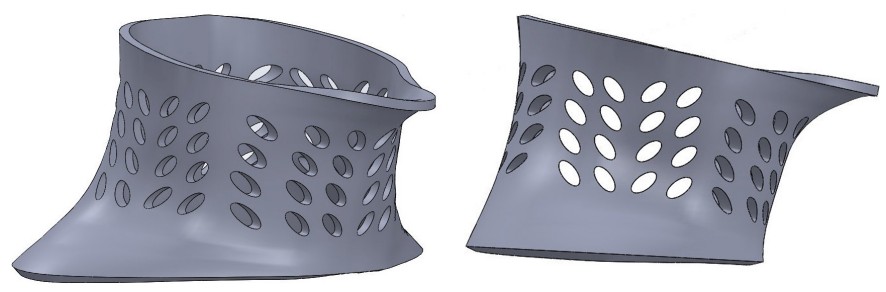

Fig. 4. CAD model of the elliptical holes pattern cervical orthosis.

This geometrical pattern, made of features with different orientations, provides orthotropic properties, keeping at the same time the porosity at a low value, being 0.87 the calculated volume fraction. Therefore, the investigation of this model, intrinsically different from the previous considered, is of interest in relation with the complex combination of forces acting on the neck orthosis.

\section{Numerical Assessment of the Customized Cervical Orthoses}

The assessment of the proposed models was done by means of finite elements analysis. The numerical simulations were used to obtain a global evaluation of the proposed architectures with the different patterns also comparing their performance in relation with some main parameters. 
The composite material Hemp Bio-Plastic ${ }^{\circledR}$ (HBP), previously introduced, was chosen for the simulations. The mechanical properties were assumed as follows: elastic modulus $4420 \mathrm{MPa}$, flexural modulus $3833 \mathrm{MPa}$ and Poisson's ratio 0.32. Each solid model was imported in a FE code and meshed by using eight nodes linear brick elements. Convergence testing was performed in order to minimize the influence of mesh density on the results. Finally, a model made of 1096142 elements was obtained for the honeycomb pattern while 1041519 were the elements of the model with the elliptical holes pattern.

A cervical orthosis is generally subjected to a combination of loads given the complex structure of the cervical spine and muscles of the neck. Investigations on cervical collars available on the market to quantify their capability of restricting the cervical spine motion during flexion, extension, axial rotation, and lateral bending were made using different experimental methods and also comparative analyses were reported in literature [23, 24]. FE numerical models of the cervical spine were also documented in literature mainly developed with the aim to study their injuries due to impact or similar $[25,26]$. In this study a simplified analysis was done in order to assess the proposed architectures.

The models of the cervical orthoses were structurally analyzed under different loading conditions namely, extension, flexion and, lateral bending to simulate the action of the neck that the orthosis has to withstand.

The applied forces were derived from an experimental study [27] which reported the three-dimensional maximum moments measured during maximum voluntary contractions of neck muscles. In that study, measurements for both genders were reported; thus it was possible to extract the maximum moments applicable for this analysis. In particular, the lower limit values for women were considered, in relation with the characteristics of the volunteer subject under study, and, from these, the force to apply for each loading arrangement considered, was calculated.

The applied forces for each simulation and the boundary conditions are depicted in Fig. 5 relatively to the model with the honeycomb pattern. Analogous loads and boundary conditions were applied to the model with the elliptical holes pattern.

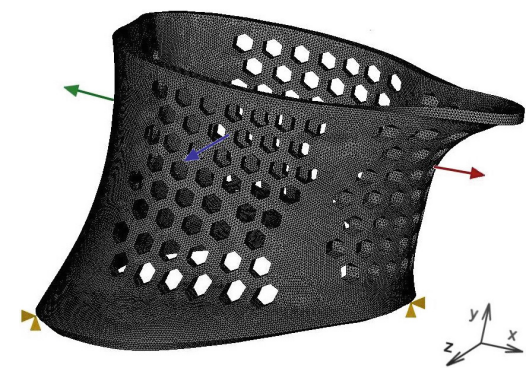

Fig. 5. FE model of the honeycomb cervical orthosis: loads and boundary conditions. 
Figure 5 shows the approximated positions of the applied forces on the outer surface of the model for clarity of the presentation, but, indeed, they were applied on the inner surface. In particular, the green arrow in the figure is relative to the load considered for extension, the red arrow is relative to flexion, while the blue arrow is relative to lateral bending. The extension force $(173.07 \mathrm{~N})$ originates a bending moment in the plane $x-y$, as well as the flexion force $(193.02 \mathrm{~N})$, while the lateral bending force $(153.84 \mathrm{~N})$ acts on the plane $\mathrm{y}-\mathrm{z}$.

The first analyzed parameter was the total displacement resulting from each loading configuration considered for both models.

Figure 6 shows the isocolour representation of the total displacement, expressed in $\mathrm{mm}$, obtained for each loading configuration, relatively to the model with the honeycomb pattern.

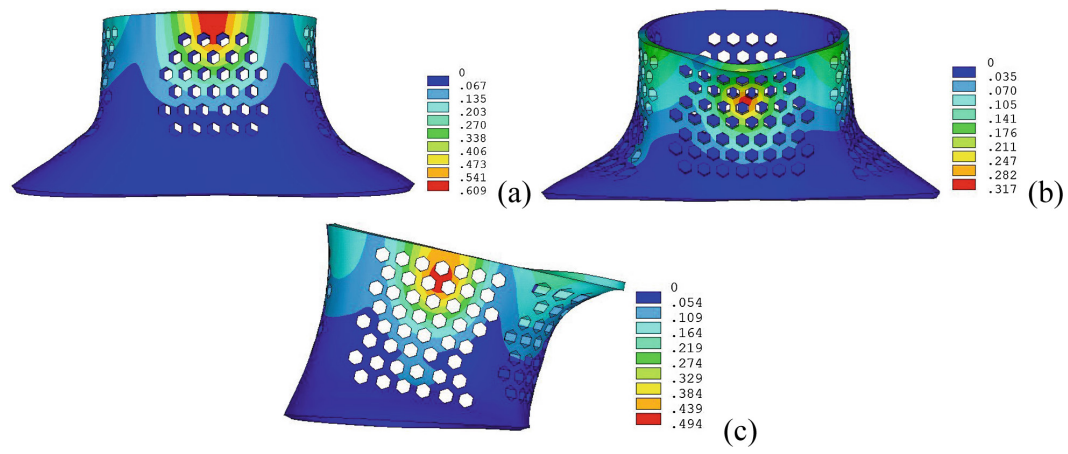

Fig. 6. Isocolour representation of the total displacement for the honeycomb pattern model and different loading configurations; (a) extension, (b) flexion, (c) lateral bending.

The figure shows that the displacements assume values that are quite limited, being under $1 \mathrm{~mm}$, for the different loading conditions. A similar distribution was obtained for the other pattern considered, as can be observed from the isocolour representation shown in Fig. 7.

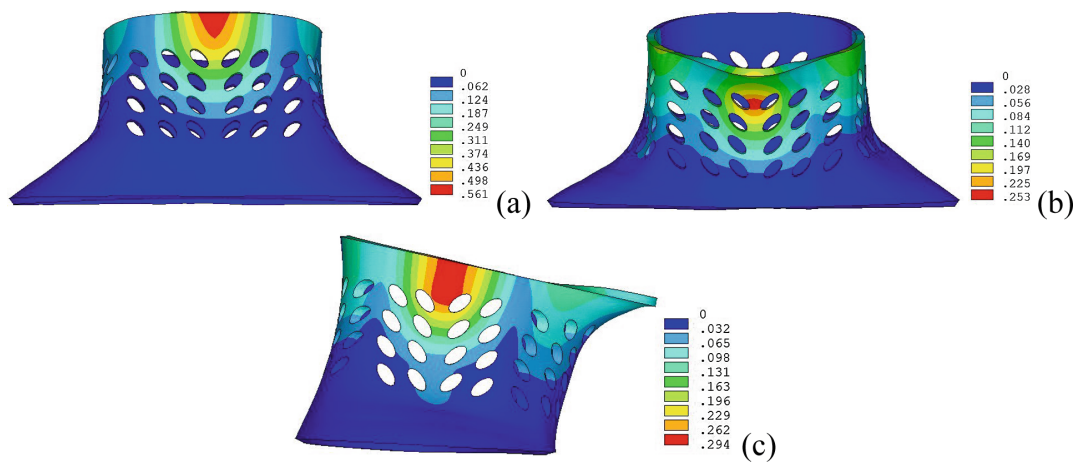

Fig. 7. Isocolour representation of the total displacement for the elliptical pattern model and different loading configurations; (a) extension, (b) flexion, (c) lateral bending. 
The results obtained for the models with the different patterns can be compared in terms of maximum total displacement, as shown in the diagram of Fig. 8.

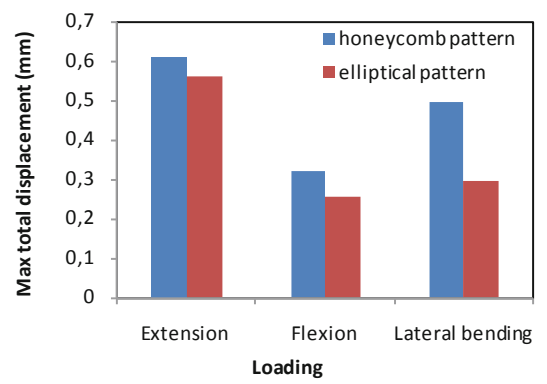

Fig. 8. Comparison between maximum total displacements of the two models obtained for different loading configurations.

From the figure it can be inferred that, for each model considered, the maximum total displacement has a similar trend and that higher values are obtained for the model of the cervical orthosis with the honeycomb pattern.

The other parameter considered for the evaluation of the two analyzed models is Von Mises stress. Figure 9 shows the isocolour representation of Von Mises stress, expressed in $\mathrm{MPa}$, obtained for lateral bending and relative to the two different geometrical patterns considered.
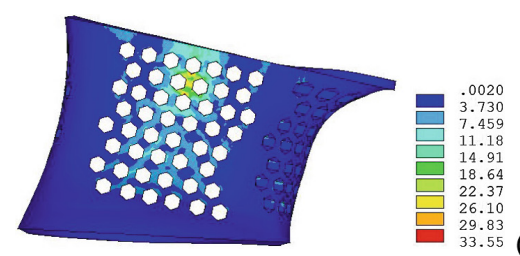

(a)

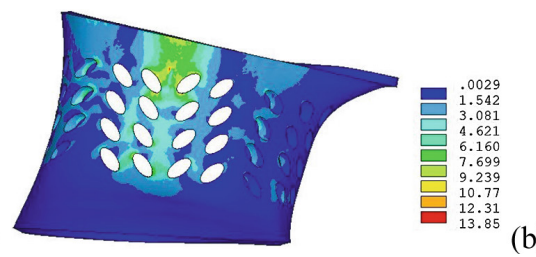

Fig. 9. Isocolour representation of Von Mises stress for lateral bending; (a) honeycomb pattern, (b) elliptical holes pattern.

From the figure it is possible to observe that Von Mises stress distribution has a similar trend for the two patterns considered. However, localized stress concentrations are more evident in the model with the honeycomb pattern with respect to the other pattern considered. Analogous results were obtained for the other loading configurations considered.

In this analysis, a parameter of relevance for the evaluation of the two patterned models is the maximum value of Von Mises stress. Figure 10 shows the values of this parameter obtained for each loading layout analyzed. 


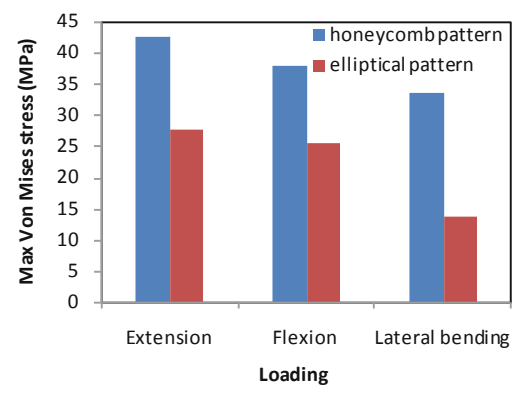

Fig. 10. Comparison between maximum Von Mises stress of the two models obtained for different loading configurations.

The diagram shows that, for each loading layout considered, higher values of the maximum Von Mises stress are obtained for the cervical orthosis model with the honeycomb pattern with respect to the elliptical pattern architecture.

However, the maximum stresses obtained for each loading configuration of the two models fall under the flexural maximum strength of HPB (69.7 MPa); accordingly, both patterns can be considered acceptable from a structural point of view.

\section{Additive Manufacturing of a Prototype Cervical Orthosis}

As a final step of this study, a prototype cervical orthosis was 3D printed. Between the two analyzed models, it was preferred to manufacture the model with the honeycomb pattern, even if it has shown a slightly global lower performance with respect to the other model assessed, since it has a lower volume fraction and this can be advantageous both for weight saving and for improved breathability.

The filament used for this case study is the HBP®, acronym standing for Hemp-bio plastic, previously introduced. The authors believe that, for its characteristic, this material could be used to create many external medical devices, besides the application proposed. In fact, it has shown many advantages, first of all in terms of mechanical properties, with a $30 \%$ of improved resistance compared to standard PLA alone, as stated by the producer [28]. This makes HBP® a good competitor with a largely used, oil-based thermoplastic, that is ABS (Acrylonitrile butadiene styrene). Furthermore, hemp shives allow to obtain lightweight composites; in particular, $\mathrm{HBP}$, thanks to its composition with hemp shives, shows an average of $20 \%$ less weight rather than standard ABS filaments [20]. The unique composition with hemp shives provide the $\mathrm{HBP} ®$ antibacterial properties [21], a feature always preferred in medicine, hence the smooth and natural superficial finish of the printed part (allowing manual minor finish for imperfections) generate an hygienic surface. The material tested has not been proven with dermal contact standards; anyhow it is being tested for many ISO standards (including skin contact) and has already obtained food contact approval. 
The prototype model was manufactured using the Printer D300 Technology® which uses the FDM process for printing. The characteristics of the printer are reported in Table 1.

Table 1. Characteristics of $3 \mathrm{D}$ printer $\mathrm{D} 300$ technology ${ }^{\circledR}$.

\begin{tabular}{l|l}
\hline Characteristic & Value \\
\hline Frame & Delta \\
\hline Print area: & $\varnothing 250 \times 300 \mathrm{~mm}$ \\
\hline Max. bed temperature & $100{ }^{\circ} \mathrm{C}$ \\
\hline Nozzle size & $0.6 \mathrm{~mm}$ \\
\hline Max. nozzle temperature & $260^{\circ} \mathrm{C}$ \\
\hline Max. Z-axis resolution & $0.1 \mathrm{~mm}$ \\
\hline X, Y, Z-axes accuracy & $0.05 \mathrm{~mm}$ \\
\hline Printer size & $380 \mathrm{~mm} \times 680 \mathrm{~mm}$ \\
\hline Power input & AC $110-220 \mathrm{~V} \mathrm{AC}, 50 / 60 \mathrm{~Hz}$ \\
\hline
\end{tabular}

Figure 11 shows the 3D printer and the prototype during an intermediate stage of manufacturing, whereas Fig. 12 reports the final prototype of the neck orthosis.

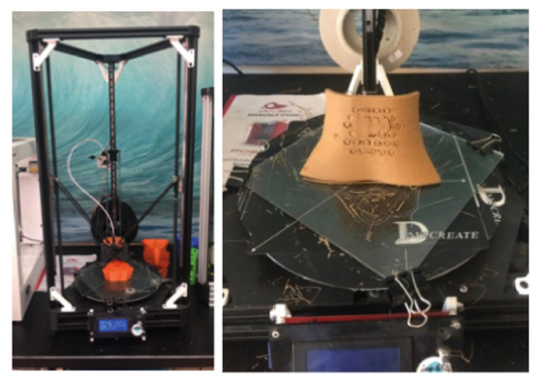

Fig. 11. An intermediate stage of the printing process.
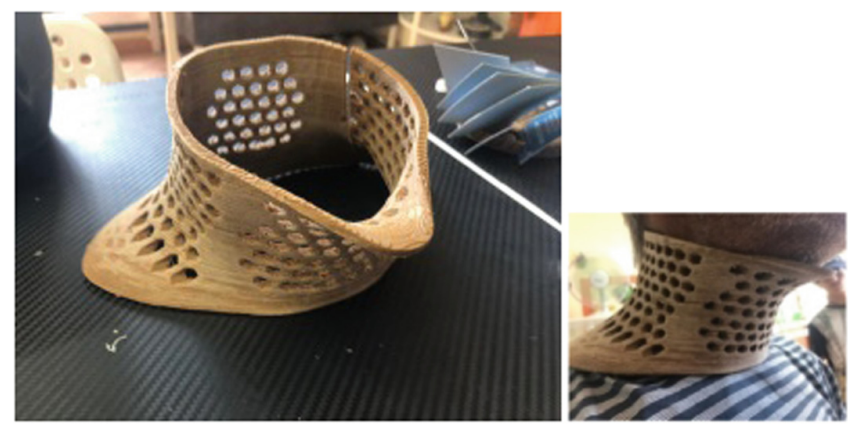

Fig. 12. Prototype of the neck orthosis. 


\section{Conclusions}

In this paper the design, assessment and manufacturing with fused deposition modelling of a customized neck orthosis are reported. The procedure of reconstruction of the neck and subsequent modelling allowed to obtain an orthosis which potentially should accomplish similar functionality to that of semi-rigid collars available on the market but with an improved comfort. In fact, the device is customized, lighter and more breathable thanks to the geometrical pattern introduced in the structure. FE numerical analysis was applied to assess two intrinsically different geometrical patterns of the modelled orthosis and the differences in terms of main structural parameters of interest for this application were highlighted. Reduced stress concentrations were also observed in the model with the elliptical holes pattern with respect the other pattern considered. Finally, the manufacturability of a prototype neck orthosis with an innovative composite material was successfully verified. The use of a bio-based material can have additional advantages including satisfying the aesthetic demands thanks to its wooden appearance. Future research will be concerned with the improvement of the procedure by automating the steps for the development of the CAD model, in order to implement the AM neck orthoses on clinical trials in orthopaedic treatments.

Acknowledgements. This research was supported in part by European Union, European Social Fund, and the University of Catania research programme 2019/2021.

\section{References}

1. Gao, W., Zhang, Y., Ramanujan, D., Ramani, K., Chen, Y., Williams, C.B., Wang, C.C.L., Shin, Y.C., Zhang, S., Zavattieri, P.D.: The status, challenges, and future of additive manufacturing in engineering. Comput.-Aided Des. 69, 65-89 (2015)

2. Ahmadi, S.M., Yavari, S.A., Wauthle, R., Pouran, B., Schrooten, J., Weinans, H., Zadpoor, A.A.: Additively manufactured open-cells porous biomaterials made from six different space-filling unit cells: the mechanical and morphological properties. Materials 8, 18711896 (2015)

3. Ambu, R., Morabito, A.E.: Porous scaffold design based on minimal surfaces: development and assessment of variable architectures. Symmetry 10(9), 361 (2018)

4. Afshar, M., Pourkamali Anaraki, A., Montazerian, H., Kadkhodapour, J.: Additive manufacturing and mechanical characterization of graded porosity scaffolds designed based on triply periodic minimal surface architectures. J. Mech. Behav. Biomed. Mater. 62, 481494 (2016)

5. Ganguli, A., Pagan-Diaz, G.J., Grant, L., Cvetkovic, C., Bramlet, M., Vozenilek, J., Kesavadas, T., Bashir, R.: 3D printing for preoperative planning and surgical training: a review. Biomed. Microdevice 20, 65 (2018)

6. Speranza, D., Citro, D., Padula, F., Motyl, B., Marcolin, F., Calì, M., Martorelli, M.: Additive manufacturing techniques for the reconstruction of 3D fetal faces. Appl. Bionics Biomech. 2017, 10 pages (2017). Article ID 9701762

7. Wong, K.C., Kumta, S.M., Sze, K.Y., Wong, C.M.: Use of a patient specific CAD/CAM surgical jig in extremity bone tumor resection and custom prosthetic reconstruction. Comput. Aided Surg. 17(6), 284-293 (2012) 
8. Pallari, J.H.P., Dalgarno, K.W., Woodburn, J.: Mass customization of foot orthoses for rheumatoid arthritis using selective laser sintering. IEEE Trans. Biomed. Eng. 57(7), 1750 $1756(2010)$

9. Lin, H., Shi, L., Wang, D.: A rapid and intelligent designing technique for patient-specific and 3D-printed orthopedic cast. 3D Print. Med. 2(4), 1-10 (2016)

10. Buonamici, F., Furferi, R., Governi, L., Lazzeri, S., McGreevy, K.S., Servi, M., Talanti, E., Uccheddu, F., Volpe, Y.: A practical methodology for computer-aided design of custom 3D printable casts for wrist fractures. Vis. Comput., 1-16 (2019). https://link.springer.com/ article/10.1007/s00371-018-01624-z

11. Kim, H., Jeong, S.: Case study: hybrid model for the customized wrist orthosis using 3D printing. J. Mech. Sci. Technol. 29(12), 5151-5156 (2015)

12. Fitzpatrick A.P., Mohanned, M.I., Collins P.K., Gibson, I.: Design of a patient specific, 3D printed arm cast. In: The International Conference on Design and Technology, KEG, pp. 135-142. DesTech Conference Proceedings (2017)

13. Kelly, S., Paterson, A., Bibb, R.J.: A review of wrist splint designs for additive manufacture. In: Proceedings of 2015 14th Rapid design, Prototyping and Manufacture Conference, Loughborough, Great Britain, pp. 1-12 (2015)

14. Cortex: Exoskeletal Cast.http://www.evilldesign.com/cortex. Accessed 15 June 2019

15. A' Design Award \& Competition, https://competition.adesignaward.com/design.phpID= 34151. Accessed 18 June 2019

16. Zhang, X., Fang, G., Dai, C., Verlinden, J., Wu, J., Whiting, E., Wang, C.C.L.: Thermalcomfort design of personalized casts. In: UIST 2012 Proceedings, Quebec City, Canada, pp. 243-254 (2017)

17. Buonamici, F., Furferi, R., Governi, L., Lazzeri, S., McGreevy, K.S., Servi, M., Talanti, E., Uccheddu, F., Volpe, Y.: A CAD-based procedure for designing 3D printable arm-wristhand cast. Comput.-Aided Des. Appl. 16(1), 25-34 (2019)

18. Blaya, F., San Pedro, P., López Silva, J., D’Amato, R., Soriano Heras, E., Juanes, J.A.: Design of an orthopedic product by using additive manufacturing technology: the arm splint. J. Med. Syst. 42(54), 1-14 (2018)

19. Prates, A.: Self-adjusting orthoses design. Scripta-Ingenia 3, 3-8 (2014)

20. Stevulova, N., Kidalova, L., Junak, J., Cigasova, J., Terpakova, E.: Effect of hemp shive sizes on mechanical properties of lightweight fibrous composites. Procedia Eng. 42, 496-500 (2012)

21. Khan, A.K., Warner, P., Wang, H.: Antibacterial properties of hemp and other natural fibre plants: a review. BioResources 9(2), 1-18 (2014)

22. Chen, C., Lu, T.J., Fleck, N.A.: Effect of imperfections on the yielding of two-dimensional foams. J. Mech. Phys. Solids 47(11), 2235-2272 (1999)

23. Gavin, T.M., Carandang, G., Havey, R., Flanagan, P., Ghanayem, A., Patwardhan, A.G.: J. Rehabil. Res. Dev. 40(6), 527-538 (2003)

24. Worsley, P.R., Stanger, N.D., Horrell, A.K., Bader, D.L.: Investigating the effects of cervical collar design and fit on the biomechanical and biomarker reaction at the skin. Med. Devices: Evid. Res. 11, 87-94 (2018)

25. Toosizadeh, N., Haghpanahi, M.: Generating a finite element model of the cervical spine: estimating muscle forces and internal loads. Scientia Iranica B 18(6), 1237-1245 (2011)

26. Osth, J., Mendoza-Vazquez, M., Sato, F., Swensson, M.Y.: A female head-neck model for rear impact simulations. J. Biomech. 51, 49-56 (2017)

27. Vasavada, A.N., Li, S., Delp, S.L.: Three-dimensional isometric strength of neck muscles in human. Spine 26(17), 1904-1909 (2001)

28. Kanesis. https://www.kanesis.it/products/\#filaments. Accessed 26 June 2019 